INTESTINE INFLAMMATION

\title{
The role of platelet activating factor in a neonatal piglet model of necrotising enterocolitis
}

\author{
A K Ewer, W Al-Salti, A M Coney, J M Marshall, P Ramani, I W Booth
}

Gut 2004;53:207-213. doi: 10.1136/gut.2003.024521

See end of article for authors' affiliations

Correspondence to:

Dr A Ewer, Neonatal Unit, Birmingham Women's Hospital, Edgbaston,

Birmingham B15 2TG, UK; a.k.ewer@bham.ac.uk

Accepted for publication 30 September 2003
Background and aims: Necrotising enterocolitis (NEC) is a potentially devastating disorder of preterm infants but its aetiology remains unclear. The aim of these studies was to develop a neonatal piglet model for NEC and to then use the model to investigate the role of platelet activating factor (PAF) in its pathogenesis.

Methods: Anaesthetised newborn piglets were divided into six groups: (i) controls, and groups subjected to (ii) hypoxia, (iii) lipopolysaccharide (LPS), (iv) hypoxia+LPS, (v) hypoxia+LPS and the PAF antagonist WEB 2170, and (vi) PAF. Arterial blood pressure (ABP), superior mesenteric artery blood flow (MBF), mesenteric vascular conductance (MVC), and arterial blood gases were recorded, and intestinal histology was evaluated.

Results: Exposure to LPS, hypoxia+LPS, or PAF all caused haemorrhagic intestinal lesions associated with varying degrees of intestinal injury. PAF caused a significant initial decrease in both MVC and MBF whereas hypoxia+LPS caused a significant late reduction in ABP and MBF with a trend towards a decrease in MVC. The effects of hypoxia+LPS on both haemodynamic changes and intestinal injury were ameliorated by WEB 2170.

Conclusions: Administration of hypoxia and LPS or of PAF in the neonatal piglet induces haemodynamic changes and intestinal lesions that are consistent with NEC. These effects are ameliorated by prior administration of WEB 2170, indicating an important role for PAF in the pathogenesis of NEC.
$\mathrm{N}$ ecrotising enterocolitis (NEC) is the commonest life threatening gastrointestinal emergency occurring in neonates. It can affect up to $5 \%$ of neonatal intensive care admissions and has a mortality of $20-40 \%,{ }^{1-3}$ a figure that has not fallen despite overall improvements in neonatal intensive care and survival. ${ }^{4-6}$ In addition to a high mortality rate, NEC is associated with significant long term morbidity due to the consequences of the disease itself, ${ }^{7}$ and also complications of prolonged parenteral nutrition, multiple surgical procedures, and short gut syndrome. ${ }^{8}$ Despite these devastating statistics, the pathogenesis of NEC remains unclear and there are no specific therapies.

Over the past 20 years, many potential aetiological factors have been postulated and subsequently refuted. However, at least one of four clinical risk factors is almost invariably present: prematurity; enteral feeding; hypoxia/ischaemia; and intestinal bacterial colonisation. ${ }^{139}$ It seems likely that these factors act singly or in concert, but whatever the apparent initiating factor, the subsequent pathology of NEC is similar, suggesting that they may trigger the same final common pathway. ${ }^{210}$

Recently, experimental evidence has led to the proposal that inflammatory mediators, particularly platelet activating factor (PAF), play an important role in this final common pathway and are crucial in the pathogenesis of NEC. ${ }^{11-14}$ PAF is an endogenous phospholipid mediator which has potent proinflammatory and biological actions. ${ }^{15}{ }^{16}$ Release of PAF is triggered by various stimuli, including hypoxia and endotoxaemia. ${ }^{17}$ PAF has a short half life in vivo, ${ }^{16}$ and is rapidly degraded by the PAF specific enzyme acetylhydrolase. ${ }^{16}{ }^{17}$ However, PAF production can be upregulated by a positive feedback loop, and it can also stimulate the production of other proinflammatory substances ${ }^{18}$ Thus once PAF production is initiated its effects can be long lasting and diverse.

In studies on adult rats, exogenous PAF produced profound, sustained, systemic hypotension with subsequent intestinal necrosis comparable with NEC; this effect was exacerbated when it was given with endotoxin. ${ }^{11}$ Further work in the adult rat showed that systemic hypoxia, ${ }^{14}$ endotoxin, ${ }^{12}$ and the combination of both insults ${ }^{13}$ led to increased production of endogenous PAF and intestinal injury which was ameliorated by prior administration of PAF antagonists. ${ }^{13}{ }^{14}$ A neonatal rat model of NEC has also been developed, using a combination of artificial feeding, bacterial colonisation of the gut, and repeated asphyxia. ${ }^{19}$ In this model, intestinal lesions were significantly reduced when PAF antagonists were given simultaneously with the experimental insults. ${ }^{20}$

However, the lack of similarity between human and rodent gastrointestinal anatomy and physiology means that extreme caution should be applied when extrapolating data from the rat. We therefore considered that a model of NEC induced in the neonatal piglet is likely to be more relevant to the human condition because of the many anatomical and physiological similarities between piglets and human neonates..$^{21}$ Importantly, when comparing the neonatal gut, piglets and humans are very similar in relation to their size, degree of maturation at birth, and anatomical and histological configuration of the gastrointestinal tract. ${ }^{21} 22$ This has led to swine being described as the best animal among common laboratory species for creating a physiological model for human infants, ${ }^{21}$ particularly when investigating the gastrointestinal tract. $^{22}$

Abbreviations: NEC, necrotising enterocolitis; PAF, platelet activating factor; LPS, lipopolysaccharide; ABP, arterial blood pressure; PSA, porcine serum albumin; $M B F$, mesenteric blood flow; MVC, mesenteric vascular conductance; SMA, superior mesenteric artery; WBC, white blood cell 
The aim of the present study was therefore to develop a model for NEC in the neonatal piglet and to investigate the potential role of PAF in its pathogenesis.

\section{METHODS \\ Animals}

Twenty seven full term neonatal Canberra White piglets were used. Their median (range) age at the time of study was 3 days (2-6) and weight $1997 \mathrm{~g}$ (1465-2270). All experiments were conducted according to the Home Office (UK) Animal (Scientific Procedures) Act (1986). Animals were nursed normally by the sow until 2-3 hours before the beginning of the experiment. In each animal, anaesthesia was induced and maintained as described by Elnazir and colleagues. ${ }^{23}$

\section{Operative protocol}

Briefly, 3-5\% halothane (Rhodia Organique Fine Ltd, Avonmouth, UK) in oxygen was delivered at $3.5 \mathrm{l} / \mathrm{min}$ initially into an anaesthetic box and then via a facemask. After induction of anaesthesia, the animal was intubated and anaesthesia was maintained with $2.5 \%$ halothane in $\mathrm{O}_{2}$ while the jugular vein was cannulated. Inhalation anaesthesia was then discontinued and anaesthesia was maintained with an intravenous infusion of Saffan (Pitman-Moore, Uxbridge, UK). Initially, a priming dose of $4-8 \mathrm{mg} / \mathrm{kg}$ Saffan diluted with normal saline to $4 \mathrm{mg} / \mathrm{ml}$ was given in small boluses. Then, Saffan was given in the same concentration by continuous intravenous infusion at $16-24 \mathrm{mg} / \mathrm{kg} / \mathrm{h}$ during surgery and at $7-12 \mathrm{mg} / \mathrm{kg} / \mathrm{h}$ during the experimental period. Saffan is a short acting steroid agent that can be given as a continuous infusion so as to achieve a stable level of anaesthesia with analgesia. This agent was chosen because it preserves central neural control of the cardiovascular system more effectively than other commonly used anaesthetics. ${ }^{23}$ Once anaesthesia was established and throughout the experiment, rectal temperature was continuously monitored and maintained at $38.5-39.5^{\circ} \mathrm{C}$ by means of heat pads beneath and heat lamps above the animal.

Respiratory variables were recorded by means of a respiratory flow head attached to the end of the endotracheal tube and connected to an electrospirometer allowing respiratory frequency and tidal volume to be recorded continuously. Pulsatile arterial blood pressure (ABP) was recorded from a cannula in a femoral artery and thereby heart rate was derived. A femoral vein was also cannulated and the catheter advanced into the inferior vena cava to allow central venous administration of drugs.

A laparotomy was then performed via a midline incision. A branch of the cranial (superior) mesenteric artery (SMA) was identified and the SMA was isolated by careful dissection. A 5 or $6 \mathrm{~mm}$ circumference cuff-type electromagnetic flow probe (Carolina Systems Inc., King, North Carolina, USA) was then placed around the SMA for continuous recordings of mesenteric blood flow (MBF). The flow probe was zeroed by temporarily occluding the vessel. Bowel handling was kept to a minimum and exposed bowel was covered in gauze swabs soaked in warm $\left(38^{\circ} \mathrm{C}\right)$ saline. Once the flow probe was in situ, the bowel was returned to the abdominal cavity and the incision closed.

Following abdominal closure, a stabilisation period of 60 minutes took place.

All physiological variables were sampled at $40 \mathrm{~Hz}$ and acquired onto a PowerMac $4400 / 160$ by a MacLab/8e System (AD instruments Ltd, Oxon, UK). Mesenteric vascular conductance (MVC) was calculated in real time by division of MBF by $\mathrm{ABP}$.

\section{Experimental protocols}

The animals were divided into six groups. The experimental protocol commenced after the stabilisation period when all physiological recordings were stable and comprised three 45 minute periods.

\section{Development of model for NEC}

Group 1 (control group; $\mathrm{n}=4$ ) spontaneously breathed room air throughout and received an intravenous bolus injection of $1 \mathrm{ml} / \mathrm{kg}$ of porcine serum albumin (PSA) via a central vein at the start of the experimental period (T0).

Group 2 (hypoxia group; $\mathrm{n}=3$ ). These animals breathed room air for the first 45 minutes from T0. This was followed by a 45 minute period of breathing $10 \%$ oxygen and then by a 45 minute period breathing room air.

Group 3 (endotoxin (lipopolysaccharide (LPS)) group; $\mathrm{n}=4$ ) spontaneously breathed room air throughout and received a bolus of LPS $(2 \mathrm{mg} / \mathrm{kg})$ via the femoral vein at T0.

Group 4 (hypoxia/endotoxin group (hypoxia+LPS); $\mathrm{n}=6$ ) followed the same protocol as group 2 but also received a dose of LPS at T0 as in group 3.

\section{The role of PAF}

Group 5 (hypoxia+LPS+WEB group; $\mathrm{n}=5$ ) received LPS and hypoxia as in group 4 . However, this group received $10 \mu \mathrm{g} / \mathrm{kg}$ of the PAF antagonist WEB 2170 via the femoral vein, 10 minutes prior to T0. This dose was chosen based on previously described therapeutic activity ${ }^{24}$ and following preliminary experimentation.

Group 6 (PAF group; $\mathrm{n}=5$ ) spontaneously breathed room air throughout and received an intravenous bolus injection of $5-7.5 \mu \mathrm{g} / \mathrm{kg}$ PAF at T0. The dose range for PAF chosen was comparable with that used in the rat model of NEC. ${ }^{11}$ The first animal received $5 \mu \mathrm{g} / \mathrm{kg}$ and the next four animals received $7.5 \mu \mathrm{g} / \mathrm{kg}$.

\section{Blood and tissue sampling}

Arterial blood samples were taken at T0 and at regular intervals throughout the experiment. These were analysed for $\mathrm{pH}$ and arterial $\mathrm{PO}_{2}$ and $\mathrm{PCO}_{2}\left(\mathrm{P}_{\mathrm{a}} \mathrm{O}_{2}\right.$ and $\mathrm{P}_{\mathrm{a}} \mathrm{CO}_{2}$, respectively $)$ using an IL1640 analyser (Instrumentation Laboratories, Warrington, Cheshire, UK) and for peripheral blood count using a MaxM Coulter counter (Beckman Coulter, USA). All experiments were terminated 135 minutes after T0 and the animals were killed by a lethal dose of pentobarbitone sodium. The thoracic and abdominal organs were removed immediately. The lumen of the gastrointestinal tract from the oesophagus to the rectum was perfused with $10 \%$ neutral buffered formalin, and then all organs placed in buffered formalin until pathological examination was performed. After fixation, the bowel was examined externally for gross lesions, particularly petechial or frank haemorrhage. Serosal lesions were identified and measured. Tissue samples were taken by one pathologist: biopsies of the serosal lesions were taken and serial biopsies were also taken at 10-15 cm intervals throughout the small and large bowel. In all cases, samples were also taken from the liver, kidneys, lungs, heart, and stomach. Sections of $4 \mu \mathrm{m}$ thickness were cut and stained with haematoxylin and eosin.

Sections were then examined by two pathologists who were blinded to the study group. Histological assessment of the most severe intestinal injury was made in each case and given a score of 0-4: 0-no significant abnormality; $1-$ mild abnormality; 2-moderate abnormality; 3-severe abnormality (localised); and 4-severe abnormality (generalised) (see table 1). 


\begin{tabular}{|ll|}
\hline Table 1 Definition of intestinal injury grading \\
\hline Grade & Histological findings \\
\hline $\begin{array}{l}\text { 0-No significant } \\
\text { abnormality }\end{array}$ & $\begin{array}{l}\text { Congestion, oedema, very occasional } \\
\text { intravillous neutrophils, occasional } \\
\text { apoptosis, hyperplastic lymphoid follicles. } \\
\text { Superficial mucosal erosion, patchy mucosal } \\
\text { haemorrhage, prominent germinal centres } \\
\text { with apoptosis and intrafollicular } \\
\text { haemorrhage, focal, acute inflammatory } \\
\text { infiltrate within the lamina propria, cryptitis, } \\
\text { and crypt abscesses. } \\
\text { Mucosal ulceration, mucosal/submucosal } \\
\text { haemorrhage, intact muscularis propria. } \\
\text { Mucosal ulceration, focal transmural } \\
\text { haemorrhage involving muscularis propria } \\
\text { and serosa. } \\
\text { Mucosal ulceration and diffuse transmural } \\
\text { haemorrhage involving muscularis propria } \\
\text { and serosa. }\end{array}$ \\
3-Severe (localised) & \\
\hline 4-Severe (generalised)
\end{tabular}

\section{Details of drug preparation}

(i) PSA was made up fresh with saline on the morning of the experiment to a concentration of $2.5 \mathrm{mg} / \mathrm{ml}$ and was stored on ice until use.

(ii) PAF (1-O-alkyl-2-acetyl-sn-glycero-3-phosphocholine; Sigma-Aldrich, Gillingham, UK) was made up from the lyophilised form with ethanol to a concentration of $2 \mathrm{mg} / \mathrm{ml}$. This solution was then divided into $100 \mu \mathrm{g}$ aliquots $(50 \mu \mathrm{l})$ and stored at $-70^{\circ} \mathrm{C}$ in Eppendorf tubes. On the day of the experiment, fresh solutions of PAF were made up with PSA after thawing and drying the PAF in nitrogen gas. Prior to use, PAF was dissolved in PSA to a concentration of $7.5 \mu \mathrm{g} / \mathrm{ml}$. (iii) LPS from Escherichia coli serotype 026:B6 (Sigma-Aldrich) was made up to a concentration of $4 \mathrm{mg} / \mathrm{ml}$ by dissolving in freshly prepared saline.

(iv) WEB 2170 (Bepafant; 5-(2-chloro-phenyl)-3,4-dihydro10-methyl-3-[(4-morpholinyl) carbonyl]-2H, 7H-cyclopen$\operatorname{ta}(4,5)$ thieno $[3,2-f][1,2,4$ triazolo- $[4,3-a][1,4]$ diazepine $]$ ) (a generous gift from Bayer AG, Leverkusen, Germany) was diluted to $60 \mu \mathrm{g} / \mathrm{ml}$ in sterile deionised water. All solutions were stored on ice until used and were brought to room temperature before injection.

\section{Statistical analyses}

All physiological data were subsequently analysed by taking the mean of 30 second samples of the data recorded on the MacLab at regular intervals, and are expressed as mean (SEM). Data from each experimental group were analysed by repeated measures and factorial ANOVA with Bonferroni post hoc tests using the Statview computer software package (version 5; SAS Institute, Cary, North Carolina, USA) in order to test differences from baseline and between groups, respectively. In all cases, $\mathrm{p}$ values of $<0.05$ were taken to be significant.

\section{RESULTS}

The laparotomy was well tolerated by all animals. Physiological variables remained stable during the surgical procedure and the subsequent 60 minute postoperative stabilisation period.

\section{Haemodynamic variables}

In the control group (group 1), all physiological variables recorded remained stable over the experimental period (see figs 1,2 ). In the other groups, the following were noted.

\section{Blood pressure}

In the hypoxia group (group 2), there was no change in blood pressure throughout the experiment. However, in the group exposed to LPS (groups 3), there was a significant fall in mean $\mathrm{ABP}$ at 45 and 90 minutes $(\mathrm{p}<0.01)$ but this recovered to baseline by the end of the experiment. In the hypoxia+LPS group (group 4), there was a moderate but non-significant fall in ABP towards the end of the experiment (see fig 1 ) but in the hypoxia+LPS+WEB (group 5), mean ABP did not alter significantly. In the PAF group (group 6), there was a brief period of hypotension (lasting only 30 seconds) immediately after administration of PAF. Brief bradycardia and apnoea were also noted over this time period.

\section{Mesenteric blood flow}

In the hypoxia and LPS group (groups 2 and 3), there was no significant change in MBF. By contrast, MBF fell steadily in the hypoxia+LPS group (group 4) and was significantly reduced at 90 and 135 minutes $(\mathrm{p}<0.01$ and $\mathrm{p}<0.001$, respectively). However, in the hypoxia+LPS+WEB group (group 5), there was no significant change in MBF (fig l).

In the PAF group (group 6), there was an immediate fall in MBF following injection of PAF and this remained significantly depressed at approximately $30 \%$ of baseline for at least 15 minutes $(p<0.001)$, gradually returning to baseline over the following 50 minutes. The fall in MBF is attributable to a decrease in MVC (see fig 1).

\section{Blood gas analysis}

Arterial blood gas analysis $\left(\mathrm{P}_{\mathrm{a}} \mathrm{O}_{2}, \mathrm{P}_{\mathrm{a}} \mathrm{CO}_{2}\right.$, and $\left.\mathrm{pH}\right)$ remained stable in the control group throughout the experiment.

All groups subjected to hypoxia (groups 2, 4, and 5) developed similar degrees of hypoxaemia during the 45 minute period of breathing $10 \%$ oxygen. In these groups, $\mathrm{P}_{\mathrm{a}} \mathrm{O}_{2}$ fell from $68(2.0) \mathrm{mm} \mathrm{Hg}$ at the start of the experiment to 40 (2.0) $\mathrm{mm} \mathrm{Hg}$ at 90 minutes; mean $\mathrm{P}_{\mathrm{a}} \mathrm{O}_{2}$ in these groups recovered to $68(6.3) \mathrm{mm} \mathrm{Hg}$ after the period of hypoxia. There was no significant difference in $\mathrm{P}_{\mathrm{a}} \mathrm{O}_{2}$ measurements between these hypoxic groups at any time during the experiment.

$\mathrm{P}_{\mathrm{a}} \mathrm{CO}_{2}$ measurements in all groups remained essentially stable throughout the experiment with no significant difference between groups at any time.

Baseline $\mathrm{pH}$ in all groups was 7.40 (0.01). In the control and hypoxia groups (groups 1 and 2), pH tended to increase towards the end of the experiment (fig 2).

There was a non-significant fall in $\mathrm{pH}$ in the LPS group (group 3) but in the hypoxia+LPS group (group 4) there was a fall in mean $\mathrm{pH}$, such that $\mathrm{pH}$ was significantly lower than controls at 90 minutes $(p<0.01)$ but recovered slightly at the end of the experiment. This effect was ameliorated to some extent in the hypoxia+LPS+WEB group (group 5, fig 2). The PAF group (group 6) developed a metabolic acidosis after 15 minutes, with a fall in mean $\mathrm{pH}$ from 7.43 (0.01) to 7.29 (0.04) $(\mathrm{p}<0.01)$ which was sustained until 45 minutes but recovered by 90 minutes (fig 2 ).

\section{Haematological analyses}

Compared with controls, over the duration of the experiment the following results were obtained: (i) there was no significant change in haemoglobin in any of the groups; (ii) there was a significant fall in white blood cell (WBC) count in the groups who received endotoxin (groups 3, 4, and 5; $\mathrm{p}<0.001$ ) but no significant change in the hypoxia and PAF groups (groups 2 and 6). The observed fall in WBC occurred as a result of neutropenia; (iii) there was a modest but statistically significant fall in the platelet count for groups 2 , 3,4 , and $5(\mathrm{p}<0.05)$ but not for the PAF group (group 6). The 

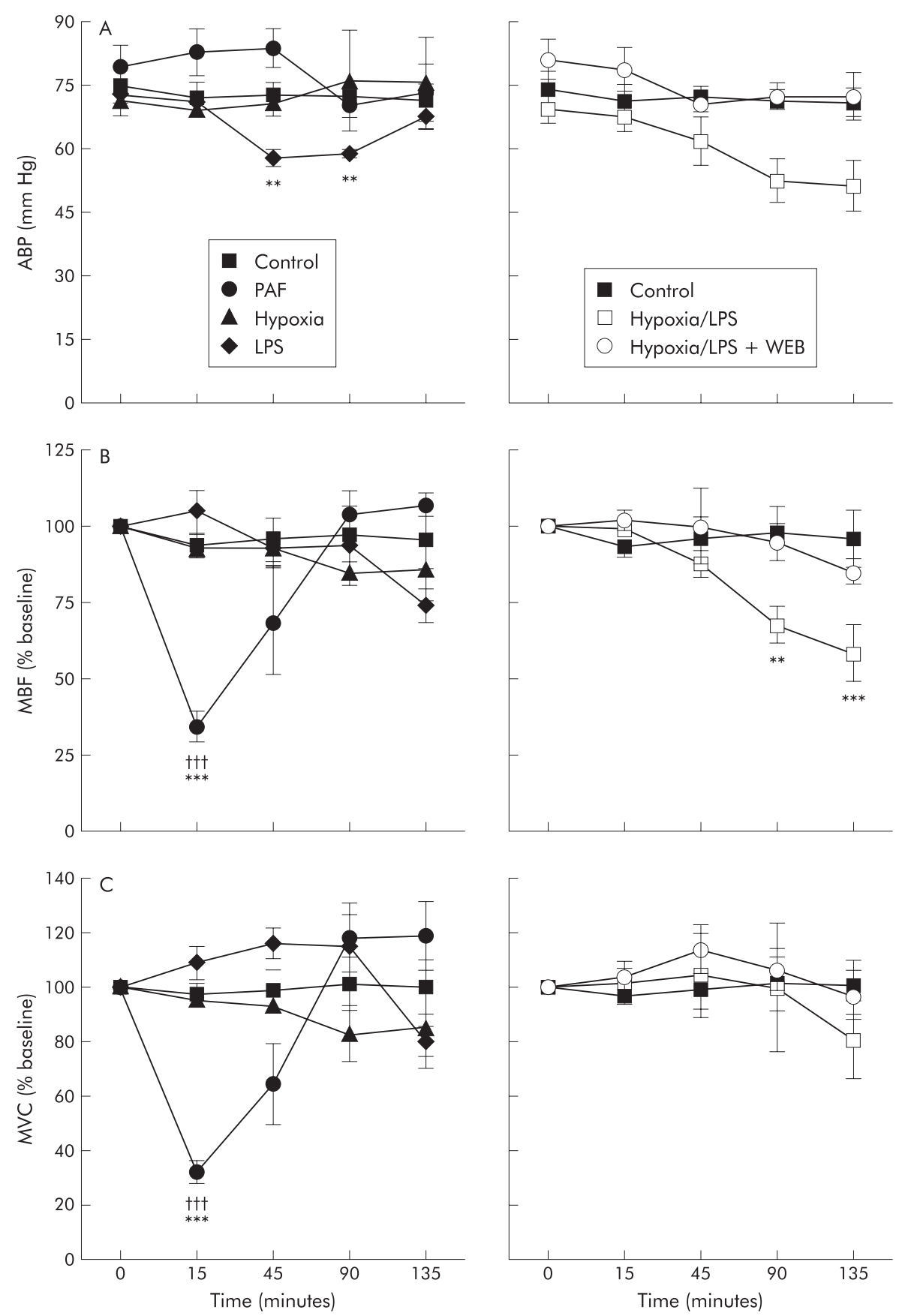

Figure 1 Physiological parameters for the study groups. (A) Arterial blood pressure (ABP); (B) mesenteric blood flow (MBF); and (C) mesenteric vascular conductance (MVC). Significant difference between the value recorded and the control group: $\dagger+\dagger p<0.001$. Significant difference between the value recorded and the baseline value for that group: ${ }^{* *} \mathrm{p}<0.01,{ }^{* * *} \mathrm{p}<0.001$. PAF, platelet activating factor; LPS, lipopolysaccharide; WEB, WEB 2170 .

platelet count was never $<350 \times 10^{9} / 1$ in any of the groups at any time

\section{Pathological analyses}

Macroscopic and microscopic examination showed that the bowel from both the control group and the hypoxia group (groups 1 and 2) were normal (table 2). Macroscopic examination of the LPS, hypoxia+LPS, and PAF groups (groups 3, 4, and 6) revealed that when compared with controls, the serosal surface of the small and large intestine had a uniform dusky discolouration, and scattered haemorrhagic lesions from 0.1 to $10 \mathrm{~cm}$ in length were present: the majority were localised to the ileum and caecum. Microscopic examination of intestine from these groups revealed a spectrum of the pathological features described in table 1 . Lesions were more severe in the PAF group and included evidence of transmural haemorrhagic necrosis. When comparing bowel injury in groups 3 and 4, LPS alone tended to cause discrete localised haemorrhagic lesions whereas hypoxia+LPS resulted in more widespread haemorrhage. There was no evidence of thrombus formation in any of the specimens.

WEB 2170 had a powerful protective effect with respect to the effects of hypoxia+LPS. In contrast with the marked 

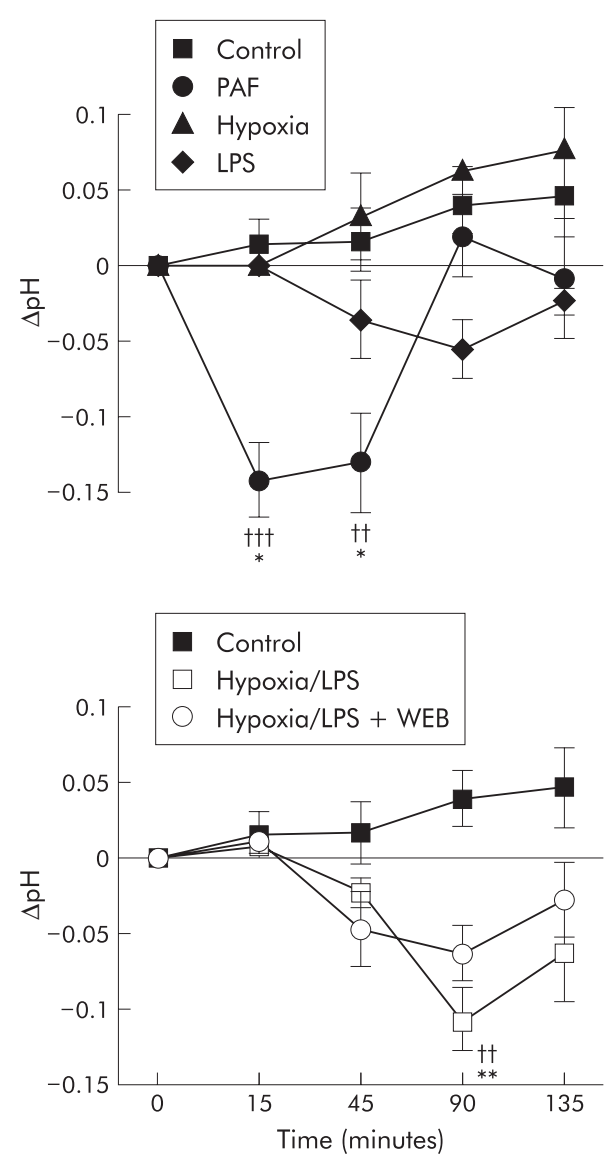

Figure 2 Change in arterial $\mathrm{pH}$ for all study groups. Significant difference between the value recorded and the control group: $t \mathrm{p}<0.01, \dagger_{\dagger} \mathrm{p}<0.001$. Significant difference between the value recorded and the baseline value for that group: ${ }^{*} p<0.05,{ }^{* *} p<0.01$, ${ }^{* * *} p<0.001$. PAF, platelet activating factor; LPS, lipopolysaccharide; WEB, WEB 2170.

macroscopic and histological changes seen in the hypoxia+LPS group, the surface of the gut appeared normal in the hypoxia+LPS+WEB group. Indeed, all but one animal in the hypoxia+LPS+WEB group (group 5) had normal intestinal histology. In this exceptional animal there was a single petechial haemorrhage $<1 \mathrm{~mm}$ in diameter-the rest of the bowel was normal.

Sections of the other major organs (heart, lungs, liver, and kidneys) were also examined microscopically and no abnormality was found in any group.

\section{DISCUSSION}

This study has demonstrated that in the neonatal piglet, the combined insults of hypoxia and endotoxin induced a reduction in $\mathrm{MBF}$, together with intestinal lesions, that were consistent with those of early NEC. These responses were ameliorated by prophylactic administration of the PAF antagonist WEB 2170, strongly implicating PAF as a causal mediator. A role for PAF in the initiation of NEC is also consistent with the observation that intravenous administration of PAF produced selective mesenteric vasoconstriction, a profound fall in MBF and, importantly, intestinal haemorrhagic necrosis, highly comparable with the later stages of NEC in the human neonate.

In the present study, systemic hypoxia alone induced minor haemodynamic effects and no changes in intestinal histology at a gross or microscopic level, while LPS induced minor hypotension and a fall in MBF with macroscopic and microscopic evidence of intestinal lesions. However, combining hypoxia with LPS markedly accentuated the haemodynamic changes, such that arterial pressure and MBF fell progressively, due in part to mesenteric vasoconstriction, and there were more widespread intestinal lesions than seen with LPS alone. In addition, this combined insult produced a significant metabolic acidosis during the experiment which may have occurred as a result of gut ischaemia. This pattern of responses seems to mimic very closely the pathophysiological process that may occur in early NEC in the human neonate. ${ }^{25}$ It should be noted that gross systemic hypotension is not a feature of the early stages of human NEC. These findings are fully consistent with the clinical experience that NEC is more likely when more than one of the common risk factors for NEC occur in concert than when singly.

It has been reported previously that intestinal lesions similar to those of NEC can be produced in the piglet only by gross insults: severe ischaemia involving ligation or occlusion of mesenteric arteries; ${ }^{26-28}$ profound asphyxia; ${ }^{29}$ or intraluminal injection of acidified casein. ${ }^{30}$ None of these experimental insults reflect conditions that occur in the clinical setting. As far as we are aware, ours is the first study to show that NEClike lesions can be produced in the neonatal piglet by combining the much more clinically plausible insults of moderate hypoxia with endotoxaemia.

Our finding that the haemodynamic responses, the metabolic acidosis, and the NEC-like lesions induced by hypoxia+LPS could be ameliorated by prophylactic administration of the PAF receptor antagonist WEB 2170, provides compelling evidence that PAF is causally involved in NEC. It is consistent with evidence obtained in adult rats that systemic hypoxia ${ }^{14}$ or endotoxin ${ }^{12}$ or a combination of the $\mathrm{tWo}^{13}$ can lead to endogenous PAF production. The combined insult of hypoxia plus LPS has been shown to act synergistically in rats with respect to hypotension, fall in $\mathrm{MBF}$, and endogenous PAF production. ${ }^{13}$ This is entirely consistent with our findings.

The proposal that PAF is a causal mediator in the effects of hypoxia plus LPS is supported by our finding that administration of exogenous PAF to the neonatal piglet also produced haemodynamic changes and intestinal lesions similar to

Table 2 Details of the experimental groups and degree of intestinal injury. The histological grade in each case is the most severe lesion seen

\begin{tabular}{llllllll}
\hline \multicolumn{7}{c}{ Histological grade } \\
\cline { 5 - 8 } Group & $\mathbf{n}$ & Mean weight $(\mathbf{g})$ & $\mathbf{0}$ & $\mathbf{1}$ & $\mathbf{2}$ & $\mathbf{3}$ & $\mathbf{4}$ \\
\hline Control & 4 & 1915 & 4 & - & - & - & - \\
Hypoxia (H) & 3 & 2385 & 3 & - & - & - & - \\
LPS & 4 & 1706 & - & 2 & - & 2 & - \\
H+LPS & 6 & 2082 & - & 4 & 1 & 1 & - \\
H+LPS+WEB & 5 & 1926 & 4 & 1 & - & - & - \\
PAF & 5 & 1990 & - & 2 & 1 & 1 & 1 \\
\hline
\end{tabular}

PAF, platelet activating factor; LPS, lipopolysaccharide; WEB, WEB 2170 . 
those of NEC. The fact that these haemodynamic responses were of a different time course and more substantial, comprising an earlier and more dramatic mesenteric vasoconstriction, and that the intestinal lesions were more severe than those induced by hypoxia plus LPS, is not unexpected. Injection of a large dose of PAF into the systemic circulation is clearly very different from the likely profile of endogenous PAF release from body tissues. Nevertheless, the observation that exogenous PAF induced strong mesenteric vasoconstriction with no significant rise in arterial pressure does indicate that the gastrointestinal tract is particularly vulnerable to its effects. The intestinal lesions induced by exogenous PAF cannot be attributed to the mesenteric vasoconstriction per se, as when the SMA of the piglet was completely occluded for 60 minutes, this resulted in detectable bowel lesions in the mucosa and submucosa, but not in the muscularis or serosal layers, ${ }^{27}$ as we observed in response to PAF.

Given that PAF has a short half life in vivo, ${ }^{16}$ it is probable that the exogenous PAF we administered stimulated endogenous PAF production via a positive feedback loop ${ }^{31}$ and that both the exogenous and endogenous PAF stimulated the production of further inflammatory mediators. ${ }^{18}$ The combined effect of all of these mediators would then explain the development of the more severe intestinal necrosis with features characteristic of the later stages of NEC. We now hypothesise that if the intestinal lesions induced by hypoxia plus LPS were initiated by endogenous PAF as we have proposed, the whole process would then have been fuelled by the influence of further PAF generated by positive feedback and other cytokines. According to this hypothesis, the extent of the intestinal lesions and the severity of the accompanying haemodynamic changes would depend on the severity of the precipitating insults, the magnitude of the increase in PAF generated, the time that elapses between the insults, and the time of observation, and also on natural inhibitors such as PAF acetylhydrolase that are available to limit the pathological cascade.

NEC is not seen in adults and very rarely seen in older infants: the neonate, particularly if born prematurely, appears to be uniquely susceptible to the disease. ${ }^{232}$ The gut vasculature of the newborn piglet is less well able to preserve tissue oxygenation in response to hypotension and hypoxia/ ischaemia than that of more mature piglets. ${ }^{33-36}$ Indeed, it would not be surprising if the high oxygen requirement of the gut in the postnatal period and a relative "immaturity" in the ability of the splanchnic circulation to cope with insults may make the newborn intestine more susceptible to periods of hypoxaemia or hypoperfusion. We have now shown that, when presented together, two insults that are likely to occur in the human neonate can induce pathological changes in the neonatal piglet that are very similar to those of early NEC. Furthermore, we have implicated PAF in these pathological changes: PAF with its vasoconstrictor and inflammatory effects would be expected to further compromise the susceptible intestinal vasculature leading to decreased tissue oxygenation, mucosal damage, and subsequent bacterial invasion.

We therefore propose that a very similar chain of events occurs in the human neonate in NEC. We believe that the use of these experimental insults in the neonatal piglet provides a model for the study of NEC that is superior to those involving far more severe insults applied to the neonatal rat, or piglet. ${ }^{19} 202829$ Our evidence that PAF is strongly implicated in the pathological changes induced by hypoxia and LPS in the neonatal piglet strengthens the case for further studies on the role of PAF in NEC. Receptor antagonists for PAF have been used in clinical trials in adults who have pancreatitis. ${ }^{37-39}$ There is at present no specific treatment for NEC and it remains a major cause of morbidity and mortality in newborn babies. Studies are now required to establish whether administration of a PAF antagonist can arrest the pathogenic process in the neonatal piglet when the early clinical signs of the pathology are just becoming manifest.

\section{ACKNOWLEDGEMENTS}

We are grateful to Professor Asif Ahmed for his help, Mr David Westwood, Mr Steven Johnson, and Ms Karen Willis for their excellent technical assistance, and Mr Anthony Lander for his helpful comments regarding the manuscript.

\section{Authors' affiliations}

A K Ewer, Birmingham Women's Hospital, and Institute of Child Health, University of Birmingham, Birmingham, UK

W Al-Salti, P Ramani, Department of Pathology, Birmingham Children's Hospital, Birmingham, UK

A M Coney, J M Marshall, Department of Physiology, University of

Birmingham, Birmingham, UK

I W Booth, Institute of Child Health, University of Birmingham,

Birmingham, UK

\section{REFERENCES}

1 Stoll BJ. Epidemiology of necrotising enterocolitis. Clin Perinatol 1994;21:205-18.

2 Kliegman R, Fanaroff A. Necrotising enterocolitis. N Engl J Med 1984;310:1093-103.

3 Brown EG, Sweet AY. Neonatal necrotising enterocolitis. Pediatr Clin North Am 1982;29:1149-70.

4 Uauy $R$, Fanaroff AA, Korones $S$, et al. Necotizing enterocolitis in very low birthweight infants: Biodemographic and clinical correlates. J Pediatr 1991; 119:630-8.

5 Loh M, Osborn DA, Lui K. Outcome of very premature infants with necrotising enterocolitis cared for in centres with or without on site surgical facilities. Arch Dis Child 2001:85:F114-18.

6 Caplan MS, Jilling T. New concepts in necrotizing enterocolitis. Curr Opin Pediatr 2001;13:111-15.

7 Vohr BR, Wright LL, Dusick AM, et al. Neurodevelopmental and functional outcomes of extremely low birth weight infants in the National Institute of Child Health and Human Development Neonatal Research Network, 1993-1994. Pediatrics 2000;105:1216-26.

8 Simon NP. Follow-up for infants with necrotizing enterocolitis. Clin Perinatol 1994:21:411-24.

9 Santulli T, Schullinger J, Heird W, et al. Acute necrotizing enterocolitis in infancy: A review of 64 cases. Pediatrics 1975;55:376-87.

10 La Gamma EF, Browne LE. Feeding practices for infants weighing less than $1500 \mathrm{G}$ at birth and the pathogenesis of necrotizing enterocolitis. Clin Perinatol 1994;21:271-306.

11 Gonzalez-Crussi F, Hsueh W. Experimental model of ischaemic bowel necrosis: the role of platelet-activating factor and endotoxin. Am J Pathol 1983; 1 12:127-35.

12 Hsueh W, Gonzalez-Crussi F, Arroyave JL. Platelet-activating factor: an endogenous mediator for bowel necrosis in endotoxaemia. FASEB $J$ 1987; 1:403-5.

13 Caplan MS, Kelly A, Hseuh W. Endotoxin and hypoxia-induced intestinal necrosis in rats: the role of platelet-activating factor. Pediatr Res 1992;31:428-34.

14 Caplan MS, Sun X, Hsueh W. Hypoxia causes ischaemic bowel necrosis in rats: the role of platelet-activating factor (PAF-Acether). Gastroenterology 1990;99:979-86.

15 Camussi G, Tetta C, Baglioni C. The role of platelet-activating factor in inflammation. Clin Immunol Immunopathol 1990:57:331-8.

16 Braquet $P$, Touqui $L$, Shen TY, et al. Perspectives in platelet activating factor research. Pharmacol Rev 1987;39:97-145.

17 Hanahan DJ. Platelet activating factor: a biologically active phosphoglyceride. Ann Rev Biochem 1986;55:483-509.

18 McManus LM. Pathobiology of platelet-activating factors. Pathol Immunopathol Res 1986;5:104-17.

19 Caplan MS, Hedlund E, Adler L, et al. Role of asphyxia and feeding in a neonatal rat model of necrotizing enterocolitis. Pediatr Pathol 1994;14:1017-28.

20 Caplan MS, Hedlund E, Adler L, et al. The platelet-activating factor antagonist WEB 2170 prevents neonatal necrotizing enterocolitis in rats. J Pediatr Gastroenterol Nutr 1997;24:296-301.

21 Tumbleson ME. In: Tumbleson ME, ed. Swine in biomedical research, vol 1. New York: Plenium Press, 1986:437-40.

22 Moughan PJ, Birtles MJ, Cranwell PD, et al. The piglet as model animal for studying aspects of digestion and absorbtion in milk-fed human infants. World Rev Nutr Diet 1992;67:40-113.

23 Elnazir B, Marshall JM, Kumar P. Postnatal development of the pattern of respiratory and cardiovascular response to systemic hypoxia in the piglet: the roles of adenosine. J Physiol 1996;492:573-85.

24 Heuer HO, Casals-Stenzel J, Muacevic G, et al. Pharmacologic activity of Bepafant (WEB 2170), a new and selective hetrazenopic antagonist of platelet-activating factor. J Pharmacol Exp Ther 1990;255:962-8. 
25 Coombs RC, Morgan MEl, Durbin GM, et al. Abnormal gut blood flow velocities in neonates at risk of necrotising enterocolitis. J Pediatr Gastroenterol Nutr 1992;15:13-19

26 De Boissieu D, Canarelli JP, Cordonnier C, et al. Effect of BN 50727 on pathological findings and tissue platelet-activating factor levels during ileal ischaemia in newborn piglets. J Pediatr Surg 1996;31:1675-9.

27 Papparella A, Deluca FG, Oyer CE, ef al. Ischaemia-reperfusion injury in the intestines of newborn pigs. Pediatr Res 1997:42:180-8

28 Sibbons PD, Spitz L, van Velzen D. Necrotising enterocolitis induced by local circulatory interruption of ileum in neonatal piglets. Pediatr Pathol 1992;12:1-14.

29 Touloukian RJ, Posch JN, Spencer R. The pathogenesis of ischaemic gastroenterocolitis of the neonate: selective gut mucosal ischaemia in asphyxiated neonatal piglets. J Pediatr Surg 1972;7:194-205.

30 Di Lorenzo M, Bass J, Krantis A. An intraluminal model of necrotizing enterocolitis in the developing neonatal piglet. J Pediatr Surg 1995;30:1138-42.

31 Sun X, Caplan MS, Hsueh W. Tumour necrosis factor and endotoxin synergistically activate intestinal phospholipase $A_{2}$ in mice. Role of endogenous platelet-activating factor and effect of exogenous plateletactivating factor. Gut 1994;35:215-19.

32 Beeby PJ, Jeffery H. Risk factors for necrotising enterocolitis: the influence of gestational age. Arch Dis Child 1992;67:432-5.
33 Nowicki PT, Miller CE. Autoregulation in the developing postnatal intestinal circulation. Am J Physiol 1988;254:G189-93.

34 Crissinger KD, Granger DN. Intestinal blood flow and oxygen consumption: responses to haemorrhage in the developing piglet. Pediatr Res 1989;26:102-5

35 Szabo JS, Mayfield SR, Oh W, et al. Postprandial gastrointestinal bloodflow and oxygen consumption: effects of hypoxaemia in neonatal piglets. Pediatr Res 1987;21:93-8.

36 Nowicki PT, Nankervis CA, Miller CE. Effects of ischaemia and reperfusion on intrinsic vascular regulation in the postnatal intestinal circulation. Pediatr Res 1993;33:400-4.

37 Johnson CD, Kingsnorth AN, Imrie CW, et al. Double blind, randomised, placebo controlled study of a platelet activating factor antagonist, lexipafant, in the treatment and prevention of organ failure in predicted severe acute pancreatitis. Gut 2001;48:62-9.

38 Kingsnorth AN, Galloway SW, Formela $\amalg$. Randomized, double-blind phase II trial of Lexipafant, a platelet-activating factor antagonist, in human acute pancreatitis. Br J Surg 1995;82:1414-20.

39 McKay CJ, Curran F, Sharples C, et al. Prospective placebo-controlled randomized trial of lexipafant in predicted severe acute pancreatitis. $\mathrm{Br} J$ Surg 1997;84:1239-43.

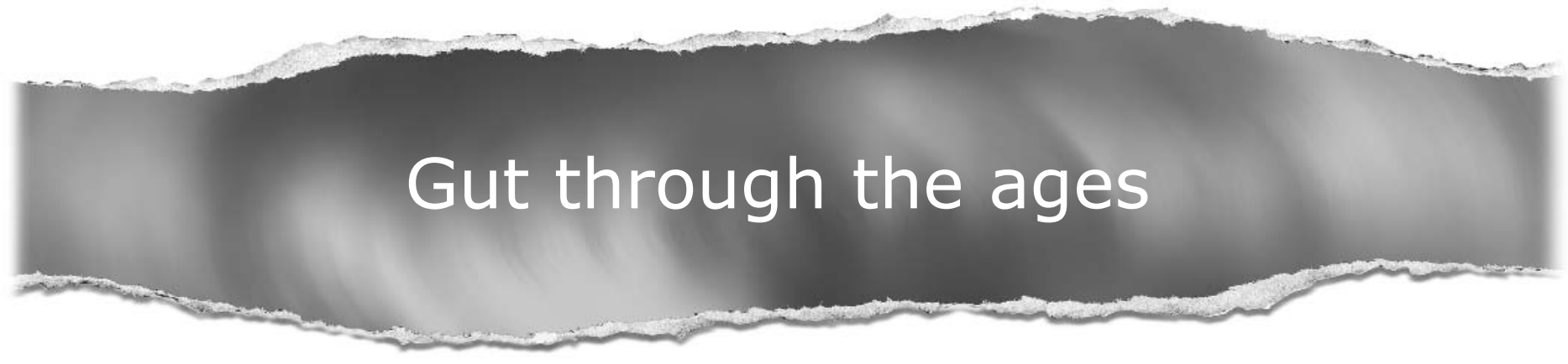

Browse the Archive

Gut online has an archive of content dating back to 1966.

Full text from 1997; abstracts from 1975; table of contents from 1966

www.gutjnl.com 